\title{
Análisis de la competencia lectora en la formación de estudiantes de bachillerato. Un estudio sobre los niveles de desarrollo logrados
}

\author{
Analysis of Reading Competence in the Formation of High School Students. A \\ Study on the Levels of Development Achieved
}

\section{Análise da competência em leitura na formação de estudantes do ensino médio. Um estudo sobre os níveis de desenvolvimento alcançados}

María Yanet Gómez Bonilla Benemérita Universidad Autónoma de Puebla, México

yanetgbonilla@yahoo.com.mx https://orcid.org/0000-0002-4278-9084

\section{Resumen}

El análisis de la competencia lectora en estudiantes de educación media superior se debe relacionar con las concepciones teóricas, las estrategias a implementar para su desarrollo, así como las propuestas de valoración sobre los desempeños que logran los educandos en los niveles de lectura literal, inferencial y crítica-analógica. El objetivo del presente trabajo fue diagnosticar la competencia lectora en alumnos de dicho nivel por ser una de las acciones comunicativas clave en su formación escolar y social. Para ello se hizo una revisión sistemática de la literatura especializada del tema de competencia lectora, con la finalidad de medir los niveles de desempeño de lectura en los educandos.

La investigación se abordó desde el enfoque cuantitativo con alcance descriptivo, porque se buscaron una serie de sucesos educativos para ser interpretados. El diseño fue no experimental transversal, ya que se aplicó una prueba de aptitud para la medición de la competencia lectora a estudiantes de quinto semestre del ciclo escolar 2017-2018 de tres subsistemas: el Centro Escolar 


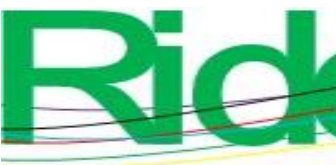

Revista Iberoamericana para la Investigación y el Desarrollo Educativo

ISSN 2007 - 7467

Pdte. Gustavo Díaz Ordaz, la preparatoria de la Benemérita Universidad Autónoma de Puebla (BUAP) y el Colegio de Bachilleres del Estado de Puebla (Cobaep). La selección de los sujetos de estudio partió de una muestra no probabilística al elegirse un subgrupo de la población derivado de las características propias de sus contextos escolares. La prueba estuvo constituida por un conjunto de preguntas que otorgaron una serie de resultados sobre el desempeño y manejo de la competencia lectora por los estudiantes de bachillerato.

Como resultado de la investigación, se encontró que los alumnos presentan varios niveles de comprensión como: 1) leer las líneas — la acción literal—; 2) leer entre líneas — la acción implícita-, y 3) leer detrás de las líneas — el punto de vista, los valores y los supuestos-. Por lo que es importante armonizar estos tres niveles de comprensión, los cuales son parte de la competencia lectora, porque cuando el estudiante domina un texto activa sus dimensiones de aprendizaje al hacer estimaciones y actuar en una situación compleja. El educando, desde la lectura, cuenta con la capacidad para generar representaciones mentales que constituyen la base del funcionamiento de esta competencia, como tema de interés en los sistemas educativos regionales, nacionales e internacionales.

Los subsistemas de educación media superior en el estado de Puebla deben impulsar estrategias que involucren a docentes y estudiantes para que aprovechen los acervos bibliográficos, así como que generen acciones que promuevan la lectura. Se trata de que los alumnos compartan momentos de estudio, consulta e investigación con propósitos muy diversos, con el fin de favorecer la interacción y el intercambio de ideas.

Palabras clave: competencia lectora, desarrollo, educación media superior, estudiantes, estudio comparativo. 


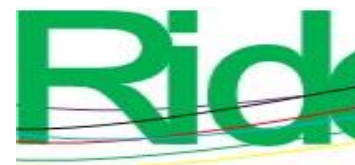

Revista Iberoamericana para la

Investigación y el Desarrollo Educativo

ISSN $2007-7467$

\section{Abstract}

The analysis of reading competence in students from upper middle education it must be related to theoretical conceptions, the strategies to be implemented for its development, as well as the evaluation proposals on the performances achieved by the students in the levels of literal, inferential and critical-analogical reading. The objective of the present work was to diagnose the reading competence in the students for being one of the key communicative actions in their school and social formation. In order to reach this, a systematic review of the specialized literature on the topic of reading competence was carried out, in order to measure the levels of reading performance into the learners.

The research was taken from the quantitative approach with a descriptive scope, because a series of educational events were searched to be interpreted. The design was not cross-sectional experimental, since an aptitude test was applied for the measurement of reading competence to students of the fifth semester of the 2017-2018 school year of three systems: the Centro Escolar Pdte. Gustavo Díaz Ordaz, the high schools of the Benemérita Universidad Autónoma de Puebla (BUAP) and the Colegio de Bachilleres del Estado de Puebla (Cobaep). The selection of study subjects was based on a non-probabilistic sample, when a subgroup of the population derived from the characteristics of their school contexts was chosen. The test was made by a set of questions that gave a series of results on the performance and management of the reading competition by the high school students.

As a result of the research, it was found that the students have several levels of understanding such as: 1) to read the lines - the literal action-; 2) to read between lines - the implicit action-, and 3) to read behind the lines - the point of view, values and assumptions-. So, it is important to match these three levels of comprehension, which are part of the reading competence, because when the student dominates a text, he activates his learning dimensions when he estimates and performs in a complex situation. The student, from reading, has the ability to generate mental representations that are the basis of the functioning of reading competence, which reaches its relevance in contemporary scenarios for being the subject of attention in regional, national and international education systems.

The upper middle education subsystems of Puebla must promote strategies that involve teachers and students in order to take advantage of the bibliographic collections, as well as generating actions that promote reading. Students are expected to share moments of consultation, research and reading with different purposes encouraging the interaction and exchange of ideas. 


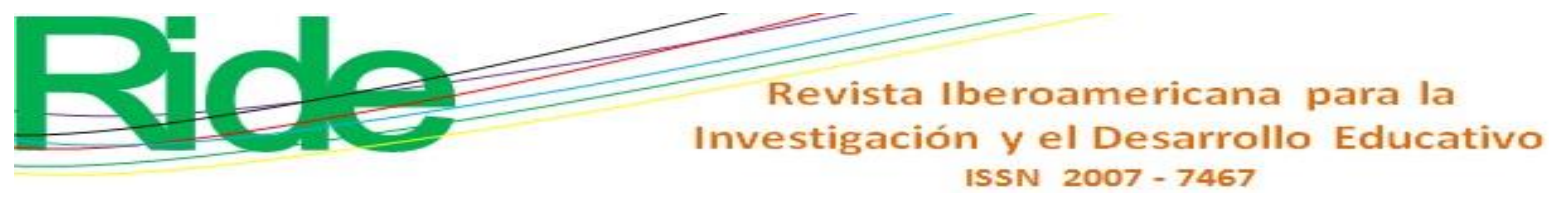

Keywords: reading competence, development, high school, students, comparative study.

\section{Resumo}

A análise da proficiência em leitura em estudantes do ensino médio deve estar relacionada às concepções teóricas, às estratégias a serem implementadas para o seu desenvolvimento, bem como às propostas de avaliação dos desempenhos alcançados pelos alunos nos níveis de leitura inferencial e literal e Crítico-analógico. O objetivo deste trabalho foi diagnosticar a competência em leitura em alunos do referido nível como uma das principais ações comunicativas em sua escola e treinamento social. Para isso, foi realizada uma revisão sistemática da literatura especializada sobre o tema competência em leitura, a fim de mensurar os níveis de desempenho em leitura em estudantes.

A pesquisa foi abordada a partir da abordagem quantitativa com escopo descritivo, pois buscouse interpretar uma série de eventos educacionais. $\mathrm{O}$ desenho foi transversal não experimental, uma vez que foi aplicado um teste de aptidão para medir a proficiência em leitura de alunos no quinto semestre do ano letivo 2017-2018 de três subsistemas: o Pdte School Center. Gustavo Díaz Ordaz, o ensino médio da Universidade Autônoma Benemérita de Puebla (BUAP) e a Escola de Bacharéis do Estado de Puebla (Cobaep). A seleção dos sujeitos do estudo foi baseada em uma amostra não probabilística, escolhendo um subgrupo da população derivado das características de seus contextos escolares. O teste consistiu em um conjunto de perguntas que forneceram uma série de resultados sobre o desempenho e o gerenciamento da proficiência em leitura por estudantes do ensino médio.

Como resultado da pesquisa, os alunos tiveram vários níveis de entendimento, como: 1) ler as linhas - a ação literal; 2) ler nas entrelinhas - ação implícita - e 3) ler nas entrelinhas - o ponto de vista, valores e premissas. Portanto, é importante harmonizar esses três níveis de entendimento, que fazem parte da competência de leitura, porque quando o aluno domina um texto, ele ativa suas dimensões de aprendizado, fazendo estimativas e agindo em uma situação complexa. O aluno, a partir da leitura, tem a capacidade de gerar representações mentais que constituem a base do funcionamento dessa competição, como um tópico de interesse nos sistemas educacionais regionais, nacionais e internacionais.

Os subsistemas do ensino médio no estado de Puebla devem promover estratégias que envolvam professores e alunos para tirar proveito das coleções bibliográficas, além de gerar ações que 


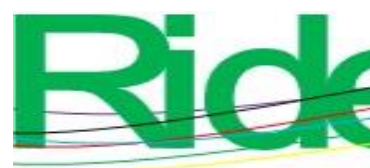

Revista Iberoamericana para la Investigación y el Desarrollo Educativo

ISSN $2007-7467$

promovam a leitura. Trata-se de estudantes que compartilham momentos de estudo, consulta e pesquisa com finalidades muito diferentes, a fim de favorecer a interação e a troca de idéias.

Palavras-chave: competência em leitura, desenvolvimento, ensino médio, estudantes, estudo comparativo.

Fecha Recepción: Junio 2019

Fecha Aceptación: Diciembre 2019

\section{Introducción}

En la actualidad se vive en una sociedad que presenta cambios significativos, provenientes de la globalización y la modernización, el avance del conocimiento científico y tecnológico; y en la cual resulta imprescindible que los individuos que la conforman cuenten con una formación sólida para adaptarse a las exigencias que el momento requiere. Así, frente a este escenario, la educación funge un papel importante —en especial en el bachillerato-, pues, una vez más, es necesario que los estudiantes cuenten con las herramientas adecuadas que les permitan hacer frente a los nuevos requerimientos de la sociedad. Hasta hace algunas décadas un determinado conjunto de conocimientos era suficiente para enfrentar y desarrollarse con éxito en la sociedad. Sin embargo, en la actualidad dichos conocimientos representan solo un fragmento para resolver algunas situaciones que se presentan en la vida diaria.

De allí que nuestro actual sistema educativo mexicano adoptara en la primera década de este siglo el enfoque por competencias. ¿El objetivo? Que todos los estudiantes que pertenecen al nivel medio superior tengan las mismas características y oportunidades al egresar del bachillerato. Es así como se manifiesta la importancia de la competencia de comunicación, específicamente la competencia lectora en el desarrollo humano de nuestros estudiantes. Por lo que se hace necesario conocer cuál es el nivel de dominio de la competencia lectora que presentan los alumnos de bachilleratos generales, colegios de bachilleres y preparatorias de la Benemérita Universidad Autónoma de Puebla (BUAP), diferentes subsistemas que pertenecen al nivel medio superior en Puebla.

En la llamada sociedad del conocimiento existen grandes organizaciones, como la Organización para la Cooperación y el Desarrollo Económicos (OCDE) y la Organización de las Naciones Unidas para la Educación, la Ciencia y la Cultura (Unesco), ocupadas en delimitar la formación de los estudiantes para cada uno de los niveles y de aplicar constantes evaluaciones internacionales y nacionales en nuestro país. Hasta el momento, los resultados han demostrado 


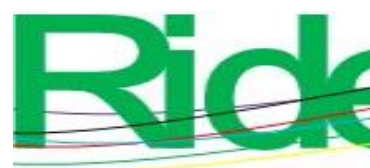

Revista Iberoamericana para la Investigación y el Desarrollo Educativo

ISSN $2007-7467$

que tienen un bajo desempeño en competencia lectora. Así lo demuestra el informe PISA 2015 (Instituto Nacional para la Evaluación Educativa [INEE], 2017), en el cual México se ubica por debajo de la media de la OCDE (493 puntos) y por encima de la media de América Latina (417 puntos) con 423 puntos. Esto demuestra que el desempeño en competencia lectora de los jóvenes de bachillerato se encuentra en el nivel dos de los seis que se presentan en el programa (INEE, 2017).

En el contexto nacional, una de las evaluaciones que se realiza cada año es el Plan Nacional para la Evaluación de los Aprendizajes (Planea). Esta evalúa los aprendizajes clave en dos áreas específicas: Lenguaje y comunicación y Matemáticas, representados en cuatro niveles de logro; el nivel IV es el más alto. Los resultados más recientes, la aplicación realizada en el año 2017, específicamente en el área de Lenguaje y comunicación, demuestran que un tercio de la población estudiantil que cursa la educación media superior se ubica en el nivel I, con 34 \%; casi uno de cada tres estudiantes se ubica en los niveles II (28\%) y III (29\%), y finalmente, casi 9 de cada 100 en el nivel IV (Secretaría de Educación Pública [SEP], 2017)

Ante este fenómeno que se presenta de manera recurrente no tan solo en el área de lenguaje sino de manera general en el desempeño académico de nuestros estudiantes, los sistemas educativos se han visto involucrados en una fase de renovación, evaluación y actualización, atendiendo y adoptando, como se ha dicho líneas atrás, el enfoque por competencias, el cual, desde la mirada de Perrenoud (2004), se define de la siguiente manera:

La aptitud para enfrentar eficazmente una familia de situaciones análogas, movilizando a conciencia y de manera a la vez rápida, pertinente y creativa múltiples recursos cognitivos: saberes, capacidades, micro-competencias, informaciones, valores, actitudes, esquemas de percepción, de evaluación y razonamiento (p. 11).

El término competencia se identifica como algo unido al saber, al saber hacer y al saber ser; traducido como el conjunto de conocimientos y de habilidades socioafectivas, psicológicas y motrices que permiten a la persona llevar adecuadamente a cabo una actividad, un papel, una función, utilizando los conocimientos, actitudes y valores que posee. Todo esto es difícil de realizar sin los recursos cognitivos que señala Perrenoud (2004), y que tienen relación con el conocimiento del individuo, su capacidad para utilizarlo y aplicarlo a situaciones concretas. 


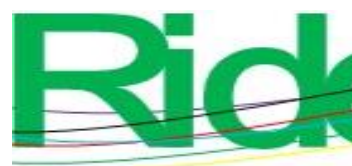

Revista Iberoamericana para la Investigación y el Desarrollo Educativo

ISSN $2007-7467$

En México, al iniciar el siglo XXI, se han hecho una serie reformas a la educación básica y media superior. El Acuerdo 444 (SEP, 21 de octubre de 2008) establece las competencias que constituyen el Marco Curricular Común del Sistema Nacional de Bachillerato, signado en el 2008, y en el cual se dividen en genéricas y disciplinarias básicas, así como las características de egreso de los estudiantes del bachillerato.

El Acuerdo 444 (SEP, 21 de octubre de 2008), asimismo, propone seis categorías para el ordenamiento de las competencias genéricas. Para efectos de la presente investigación se enfatiza en la categoría de comunicación "Se expresa y comunica", que busca desarrollar en el alumno de educación media superior la competencia genérica número cuatro: "Escucha, interpreta y emite mensajes pertinentes en distintos contextos mediante la utilización de medios, códigos y herramientas apropiados" (p. 3). En los estudiantes del nivel educativo en cuestión radica en las diversas formas en que el ser humano es capaz de comunicarse y establecer relaciones con otros y el entorno a través de la comunicación. Aunado a ello, en el artículo 6 se retoma la competencia de comunicación que tiene importante relevancia por su transversalidad en el currículo, porque es imprescindible en todas las disciplinas y espacios curriculares; además de ser transferible al reforzar la capacidad de los estudiantes de adquirir otras competencias y propiciar habilidades para comunicarse efectivamente en el español y en lo esencial en una segunda lengua en diversos contextos, mediante el uso de distintos medios e instrumentos (SEP, 21 de octubre de 2008). Quienes hayan desarrollado estas competencias podrán leer críticamente y comunicar y argumentar ideas de manera efectiva y con claridad oralmente y por escrito. Además de estar orientada a la reflexión sobre la naturaleza del lenguaje y a su uso como herramienta del pensamiento lógico (SEP, 21 de octubre de 2008).

En palabras de Dell Hymes (1971), la competencia comunicativa se relaciona con saber cuándo hablar, cuándo no, con quién, de qué y en qué forma; es decir, se trata de la capacidad de formar enunciados que no solo sean gramaticalmente correctos, sino también socialmente apropiados. Por lo tanto, el término de competencia comunicativa se comprende como un conjunto de habilidades relacionadas con la comunicación humana, esto es, referida a los conocimientos lingüísticos, sociales y culturales necesarios para que se lleve a cabo una comunicación adecuada en el marco de situaciones reales. 


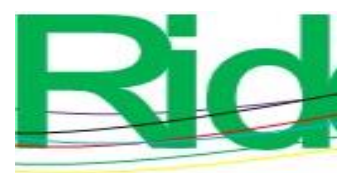

Revista Iberoamericana para la

Investigación y el Desarrollo Educativo

ISSN $2007-7467$

El desarrollo de la competencia comunicativa se logra con la suma de diversas subcompetencias, tal y como la competencia discursiva, la competencia textual, la competencia sociolingüística, la competencia lingüística o gramatical, la competencia pragmática, y para efectos de esta investigación, la competencia lectora.

\section{Competencia lectora}

La competencia lectora es elemento clave para la formación del ser humano, pues mediante ella se accede al conocimiento de todas las áreas, permite la incorporación del sujeto al contexto actual y representa el acceso a la cultura. Si se trabaja con los estudiantes el dominio de esta competencia, se asegurará el desarrollo de habilidades básicas del pensamiento.

Escamilla (2008) destaca la importancia que tienen en la actualidad las competencias de comunicación (la lectora, entre ellas) en estudios y evaluaciones que se desarrollan para determinar la calidad y eficacia de los programas y sistemas educativos. Un ejemplo claro es el Programa para la Evaluación de los Estudiantes (PISA), que se realiza desde el año 2000 y cuyo propósito es medir y analizar el desempeño de los jóvenes de 15 años en tres temáticas específicas: lectura, matemáticas y ciencias.

PISA define la competencia lectora como "la capacidad de comprender, utilizar y analizar textos escritos para alcanzar los objetivos del lector, desarrollar sus conocimientos y posibilidades y participar en la sociedad" (OCDE, 2006, p. 48). En dicha propuesta, patrocinada como se puede ver por la OCDE, se destaca, junto a la comprensión lectora, el interés y compromiso por la lectura como elemento primordial. En su conjunto, estos dos elementos son una herramienta para que los lectores puedan desarrollarse en una variedad de acontecimientos. De esta manera, se visualiza la importancia de desarrollar la competencia lectora en estudiantes de bachillerato y retomar aspectos fundamentales para su proceso como son la lectura y la comprensión de diferentes textos implicando el desarrollo de habilidades básicas. 


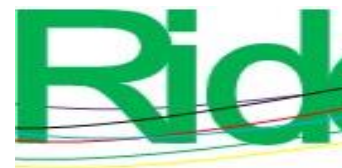

La lectura y la comprensión: procesos centrales de la competencia lectora

A lo largo del tiempo, el concepto o idea que se tenía de la lectura ha ido cambiando. En un inicio se contemplaba como la transferencia de información, y así, de esta manera, se extraía lo que el texto contenía; posteriormente diferentes estudios fueron reflejando que este proceso de leer debería ser más completo y se establecieron modelos, niveles, intereses y propósitos. De tal forma que leer implicaba, a partir de entonces, interactuar con el texto; y en ese momento el sujeto entró en contacto con el contexto desde diferentes aspectos de la vida: económicos, históricos, sociales, culturales y cotidianos mediante la comprensión y la interpretación (Solé, 1998).

Para los estudiantes de educación media superior, el desarrollo de la lectura y la comprensión de diferentes tipos de textos representa múltiples beneficios. Por ejemplo: la adquisición de la mayoría de los conocimientos o aumentar su nivel cultural; a través de ambas habilidades se transfieren conocimientos de una generación a otra; también se despierta la sensibilidad del educando al proporcionarle diversas emociones.

Diversos autores que han definido el concepto de lectura han enfatizado en la importancia de esta desde la educación inicial. Para Ferreiro (2002), la lectura es un proceso de coordinación de información de diversa procedencia, cuyo objetivo es obtener el significado expresado linguísticamente, es decir, la interpretación del texto es la asignación de significado que le da el lector a este después de una lectura convencional; mucho de su significado proviene del contexto.

Otra mirada es la que define a la lectura como un proceso interactivo entre dos elementos fundamentales: el lector y el texto, y de esta interacción se logra una apropiación que permite al sujeto construir su propio significado. En este aspecto encontramos que la lectura como proceso interactivo representa el medio que permite al sujeto otorgar un sentido personal al texto, atendiendo al conjunto de saberes y experiencias adquiridas a lo largo de la vida, y aplicarlos a un contexto determinado (Gómez, 1996, p. 311).

Desde otra mirada, Kenneth Goodman (1982) afirma que la lectura es un proceso en el que el pensamiento y el lenguaje interactúan para que el lector obtenga un sentido de lo que está escrito; por eso es un proceso constructivo, en el que importa que el lector interactúe de manera activa en el texto. El lector interpreta y construye significados literales del texto, pero también realiza inferencias que van más allá de las palabras encontradas en él. Esto es porque el sujeto atiende o refiere conocimientos propios de lugares, gente, ideas, aprendizajes escolares, eventos, etc., los cuales ha obtenido a lo largo de su vida a partir del conocimiento que tiene del mundo; a 


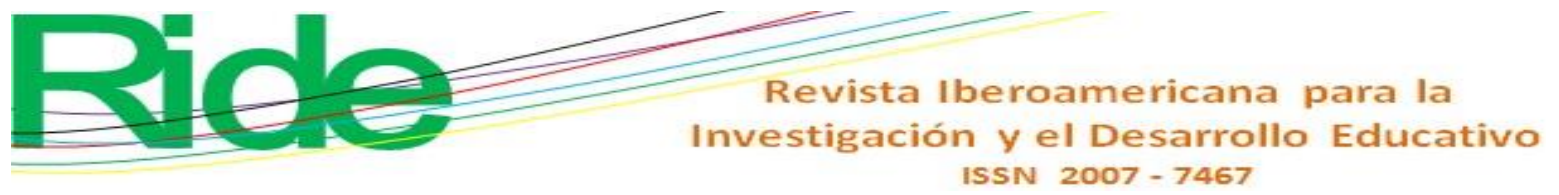

ello Emilia Ferreiro (2002) lo llama “contexto interno psicológico de redes semánticas organizadas de conocimientos del mundo que se reflejan parcialmente en el vocabulario del lector" (p. 213).

A pesar de que a través del tiempo se han presentado distintas concepciones teóricas con respecto al tema de la lectura, como el modelo ascendente, modelo descendente y el modelo interactivo, por mencionar algunos, resulta que en algunos aspectos o características no deben ser considerados excluyentes entre sí. Antes bien, se deberán abordar para enriquecer el proceso de lectura.

La comprensión lectora representa una habilidad cognitiva compleja en la cual se ven inmersos múltiples procesos (léxicos, sintácticos, semánticos) que además interactúan entre sí; de tal manera que el término corresponde a la habilidad de desarrollar significados a través del reconocimiento de ideas más importantes extraídas de un texto y la capacidad para establecer relaciones entre estas y los conocimientos o experiencias adquiridas con anterioridad. Por lo que en el ámbito educativo la lectura tiene diversos propósitos: uno de ellos es que los estudiantes adquieran el hábito de la lectura y se relacionen con la literatura, y otro que la utilicen como estrategia para aprender contenidos y conocimientos desde las diferentes áreas y ámbitos de la vida (Solé, 1998).

El logro de la comprensión lectora se da cuando el sujeto ya sabe leer y escribir y es capaz de activar conocimientos previos, interpretar el texto y tener procesos de autorregulación; resultado de sus experiencias de vida y conocimiento del mundo. La interacción que se da entre el lector y el texto es la base del proceso de comprensión, es decir, si el sujeto lector desconoce, o bien no establece relaciones difícilmente llegará a la comprensión, a la generación de nuevos conocimientos y su aprendizaje no será significativo, por lo que lo leído formará parte apenas de la memoria a corto plazo.

\section{Análisis de la competencia lectora a partir de los tres niveles de lectura}

El diagnóstico y análisis del desempeño que presentan los estudiantes de media superior en función de la competencia lectora se fundamenta en la propuesta de los tres niveles lectura de Donna Kabalen y Margarita de Sánchez (1997), quienes, de acuerdo con el paradigma de procesos, presentan la siguiente escala: 


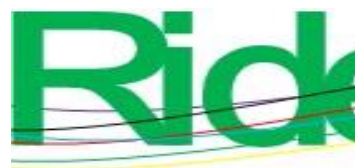

Revista Iberoamericana para la Investigación y el Desarrollo Educativo

ISSN $2007-7467$

Lectura de nivel literal consiste en obtener información dada explícitamente en el texto.

Lectura de nivel inferencial-crítico consiste en establecer relaciones entre lo que se está leyendo para suponer o inferir datos que no se dan directamente en el texto. Las inferencias pueden provenir de las suposiciones del lector o de las relaciones entre los datos que se dan directamente en el texto. Se considera que la lectura inferencial es una lectura entre líneas.

Lectura de nivel analógico-crítico consiste en relacionar lo que se decodifica directamente en el texto o lo que se infiere, con otra información extraída de otro texto o tomada de la realidad o de algún otro contexto del pasado, presente o futuro. La lectura analógica permite comparar la información y extender el conocimiento más allá de lo dado en el texto (Kabalen y Sánchez, 1997, p. 20) (cursivas añadidas).

El logro de estos niveles de lectura que presentan dichas autoras depende del desarrollo de procesos cognitivos que, con la práctica, permitirán el desarrollo de la competencia lectora analizando la información que presentan los textos.

De tal forma que en el nivel literal entran nueve procesos básicos del pensamiento: la observación, comparación, relación, clasificación simple, ordenamiento, clasificación jerárquica, análisis, síntesis y evaluación. Estos procesos tendrán relación específica con la información explícita que presentan los textos; asimismo, el desarrollo de estos procesos prepara al estudiante lector a alcanzar niveles de comprensión más complejos. La lectura de nivel literal tiene como propósito que el estudiante de media superior identifique información explícita en el texto utilizando los nueve procesos cognitivos ya especificados, es decir, toda pregunta que se le plantee a este la podrá encontrar fácilmente en alguna línea del texto.

El nivel de lectura de nivel inferencial se apoya en la lectura de nivel literal y sus nueve procesos básicos del pensamiento. Sin embargo, como era de esperarse, requiere de procesos más complejos, de una comprensión más profunda, y para ello el lector necesita desarrollar habilidades de síntesis, análisis y razonamiento. En estas se "incluyen procesos de decodificación e inferencia, de razonamiento deductivo e inductivo y de adquisición de conocimiento" (Kabalen y Sánchez, 1997, p. 97). Todo ello para interpretar mensajes implícitos y plantear inferencias que permitan, a partir de los conocimientos previos del lector, obtener información que no está presente en el texto. 


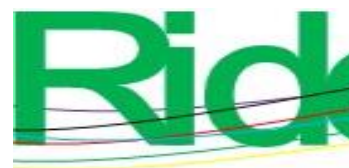

Revista Iberoamericana para la

Investigación y el Desarrollo Educativo

ISSN $2007-7467$

Finalmente, de forma análoga al caso anterior, la lectura de nivel analógico-crítico e interpretativo-crítico implica los niveles de lectura anteriores, el literal e inferencial. Y aún más: para lograr este nivel se requiere de un conjunto de procesos de pensamientos que establezcan relaciones de orden superior. De igual forma, "involucra la aplicación de cuatro procesos superiores de pensamiento: la decodificación, la inferencia, la funcionalización y la aplicación" (Kabalen y Sánchez, 1997, p. 155). Según las autoras, en este nivel el lector realiza las siguientes actividades:

a) Aplica los niveles de lectura previamente estudiados, $b$ ) interpreta las temáticas del escrito, c) establece relaciones analógicas de diferente índole, por ejemplo entre el presente, el pasado y el futuro, entre una lectura y otra, entre secuencias de sucesos que ocurren en diferentes contextos, etc., $d$ ) establece juicios acerca de lo leído, y e) formula sus propias conclusiones (Kabalen y Sánchez, 1997, p. 155).

En la actualidad, es de suma importancia que los estudiantes de bachillerato dominen los tres niveles de lectura, con el objetivo de que dicho dominio les permita insertarse en cualquier contexto y tomar decisiones ante diversas situaciones de la vida cotidiana.

\section{Método}

\section{Diseño de la investigación}

La investigación tiene un diseño cuantitativo con alcance descriptivo, porque busca el descubrimiento de hechos para continuar con la interpretación que permite valorar los resultados y aportar datos clasificados, a partir de procedimientos donde se identifican fenómenos y experiencias producto de la construcción y aplicación del instrumento. El proceso de investigación se fundamenta en el campo analítico y crítico, ya que se valora el nivel de desarrollo que presentan los estudiantes de bachillerato en relación con la competencia lectora. La investigación es de tipo no experimental transversal, puesto que se trabajó con un instrumento en la modalidad de prueba de aptitud a un grupo de sujetos en un mismo momento. 


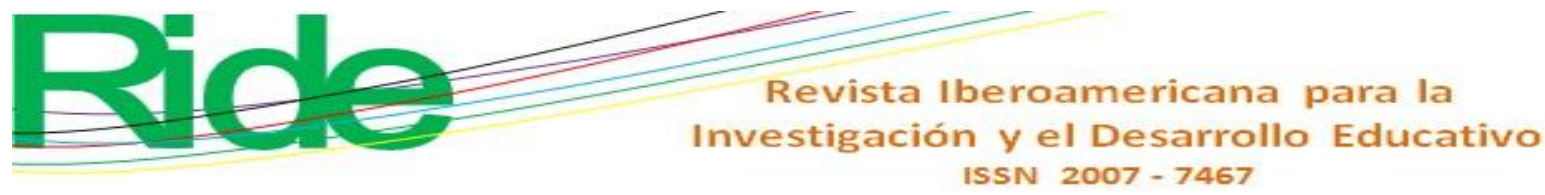

\section{Sujetos de estudio}

El instrumento se aplicó en tres subsistemas de nivel medio superior del estado de Puebla: la Preparatoria 2 de Octubre de 1968 de la BUAP, el plantel 21 del Colegio de Bachilleres del Estado de Puebla y el Centro Escolar Pdte. Gustavo Díaz Ordaz. En específico, se aplicó a estudiantes de quinto semestre del ciclo escolar 2017-2018. Al proponer la enseñanza de una serie de contenidos determinados que favorecen el desarrollo y manejo de la competencia lectora, se consideró como objeto de estudio el campo disciplinar de la lengua y la comunicación.

La selección de los sujetos de estudio corresponde a una muestra no probabilística (Hernández, Fernández y Baptista, 2014, p. 176): se eligió un subgrupo de la población estudiantil de bachillerato: 50 estudiantes de cada uno de los subsistemas arriba mencionados. El total de alumnos participantes fue de 150, por lo que la pertinencia del trabajo de campo deriva de la elección de sujetos informantes con características especificadas en el planteamiento del problema (Hernández et al., 2014, p. 190).

\section{Instrumento}

El instrumento de investigación adoptó la estructura de una prueba de aptitud, la cual se construyó de acuerdo con el enfoque formativo en el área de lenguaje dirigido a los alumnos de bachillerato. La prueba estuvo constituida por un conjunto de preguntas, de tal manera que la información que se obtuvo de ella evidenció una serie de indicadores con respecto a los niveles de desempeño y manejo de la competencia lectora por los estudiantes de bachillerato.

La prueba de aptitud es un instrumento que permite evaluar el aprendizaje de los estudiantes en el bachillerato para detectar lo correcto o incorrecto en las respuestas del alumno. Además, el docente puede valorar el comportamiento de aprendizaje en las distintas esferas del conocimiento (Bernard, 2000). Basada en un enfoque cognitivo, busca obtener registros de los saberes orientados a la competencia lectora, cuando el estudiante aporta a su propia actividad interna los dominios del lenguaje para transformar la realidad que recibe en una interpretación simbólica. La prueba permite extraer información sobre el aprendizaje del alumno, cuando describe cada determinado tiempo lo que acaba de hacer como demostración de lo que sabe con respecto a los contenidos específicos de la asignatura. 


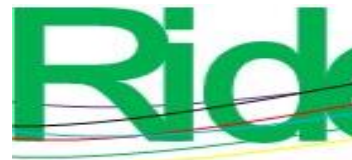

\section{Revista Iberoamericana para la Investigación y el Desarrollo Educativo \\ ISSN $2007-7467$}

La prueba de aptitud buscó obtener información sobre la competencia lectora que manejan los educandos en el nivel de bachillerato, para así generar un ejercicio comparativo que enriquezcan las dimensiones de análisis e interpretación de la investigación. La prueba constó de 16 preguntas y se estructuró en tres variables de estudio: Lectura de nivel literal, Lectura de nivel inferencial y Lectura de nivel crítico-analógica (véase tabla 1).

Tabla 1. Variables e indicadores de estudio del instrumento de investigación

\begin{tabular}{|c|l|c|}
\hline Variables de estudio & \multicolumn{1}{|c|}{ Indicadores de estudio } & Preguntas \\
\hline $\begin{array}{c}\text { Lectura de nivel } \\
\text { literal }\end{array}$ & Dominio general del tema & $1,2,8,13$ \\
\hline \multirow{2}{*}{$\begin{array}{c}\text { Lectura de nivel } \\
\text { inferencial }\end{array}$} & Dominio de lenguajes & $3,4,17$ \\
\cline { 2 - 3 } & Calidad de razonamiento & $5,9,10$ \\
\cline { 2 - 3 } & Error y su naturaleza & 14,15 \\
\hline \multirow{2}{*}{$\begin{array}{c}\text { Lectura de nivel } \\
\text { crítico-analógica }\end{array}$} & Abstracción & $6,7,11$ \\
\cline { 2 - 3 } & Conciencia cognitiva & 12,16 \\
\hline
\end{tabular}

Fuente: Elaboración propia

La prueba de aptitud para la medición de la competencia lectora en la variable Lectura de nivel literal considera al indicador "Dominio general del tema" con el propósito de valorar los conocimientos del estudiante pertinentes a la tarea y planificación de la misma. Esta variable se integra con las preguntas 1, 2, 8 y 13, las cuales miden el conjunto de conocimientos que el estudiante emplea en la tarea asignada, las ausencias o dudas en cuanto a la temática trabajada.

En la variable Lectura de nivel inferencial se aborda el indicador "Dominio de lenguajes", donde el estudiante utiliza los registros verbales y analógicos, ubicados en las preguntas 3, 4 y 17, para medir la comprensión y dominio verbal, así como la percepción estructural del pensamiento con los contenidos que organiza. Con el indicador "Calidad de razonamiento" se busca identificar la lógica que el alumno utiliza en la actividad de inferir conocimientos a partir de otros. Así, las preguntas 5, 9 y 10 representan los procesos diferenciales del participante a través de la organización de la información que se le presenta. Por otra parte, con el indicador "Error y su naturaleza" se buscan los aspectos que inciden en los fallos cometidos por el estudiante en la comprensión, planificación y ejecución de la prueba. Las preguntas 14 y 15 muestran las causas del error, así como las variedades de errores que pueden mostrar. 


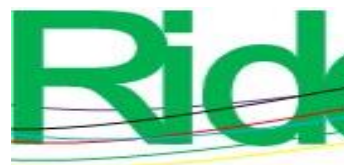

Revista Iberoamericana para la Investigación y el Desarrollo Educativo

ISSN $2007-7467$

Finalmente, la variable Lectura de nivel crítico presenta el indicador "Abstracción” con el propósito de ubicar el dominio de los contenidos que el estudiante procesa en la prueba. En las preguntas 6,7 y 11, deben responder considerando datos que no contiene la prueba y conectarlos por su cuenta con la información que se les va presentando. Igualmente, el indicador "Conciencia cognitiva" considera la percepción y procedimientos ejecutados, porque son los conocimientos que el alumno tiene sobre su propio pensar. Las preguntas 12 y 16 representan el nivel de seguridad, control de procesos y el logro de objetivos. En el instrumento también se consideran los datos generales de los estudiantes, que aportan aspectos informativos: nombre, grupo, unidad académica o escuela, edad, género.

\section{Procedimientos}

La aplicación del instrumento se llevó a cabo en tres instituciones educativas del estado de Puebla, ya especificadas anteriormente, y fue contestado por los participantes entre agosto y noviembre de 2017, quienes, además, se encontraban en la fase preparatoria de egreso para continuar con sus estudios de licenciatura, o bien ingresar al ámbito laboral. Los estudiantes en ese momento ya habían cursado las asignaturas del campo disciplinario de lenguaje y comunicación.

Una vez aplicada la prueba, se codificó la información y se creó una base de datos que permitió hacer el análisis y valoración de los resultados. Se ubicaron las respuestas que emitió cada uno de los informantes, y se utilizó para su medición una escala de desempeño. La medición de reactivos se manejó de arriba hacia abajo, en función del nivel de respuesta del estudiante (véase tabla 2). 


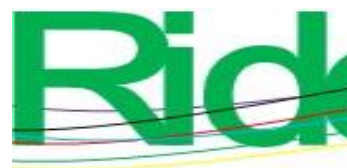

\section{Revista Iberoamericana para la Investigación y el Desarrollo Educativo \\ ISSN $2007-7467$}

Tabla 2. Escala de medición para asignar puntajes a las respuestas de la prueba

\begin{tabular}{|c|c|l|}
\hline Escala & Rango & \multicolumn{1}{c|}{ Atributos } \\
\hline 5 & Destacado & $\begin{array}{l}\text { El estudiante acierta con puntualidad la esencia de la pregunta en su } \\
\text { conjunto y en sus partes y la soluciona correcta y congruentemente en } \\
\text { todos sus grados. }\end{array}$ \\
\hline 4 & Bueno & $\begin{array}{l}\text { El estudiante responde puntualmente, por lo que no ejecuta errores de } \\
\text { relevancia, así que no se perciben vacíos en la construcción de la } \\
\text { respuesta. }\end{array}$ \\
\hline 3 & Regular & $\begin{array}{l}\text { El estudiante evidencia algunos titubeos y debilidades en la elaboración } \\
\text { de la respuesta, aunque en determinados referentes la respuesta es } \\
\text { racional y lógica. }\end{array}$ \\
\hline 2 & Suficiente & $\begin{array}{l}\text { El estudiante evidencia variedad de incertidumbres en el planteamiento, } \\
\text { proceso, desarrollo y construcción de la respuesta. }\end{array}$ \\
\hline 1 & No & $\begin{array}{l}\text { El estudiante evidencia el dominio mínimo de contenido y se muestra } \\
\text { confundido a lo largo de la generación de la respuesta. }\end{array}$ \\
\hline
\end{tabular}

Fuente: Elaboración propia

\section{Resultados}

En el presente apartado se muestra el análisis, la descripción e interpretación de los resultados que arrojó la aplicación de la prueba de aptitud, cuya finalidad, vale la pena reiterar, es la de medir, en función de la competencia lectora, el nivel de desempeño que muestran los estudiantes de diferentes subsistemas de la educación media superior en la ciudad de Puebla. En este proceso de análisis se aborda cada uno de los elementos contenidos en la prueba con el propósito de comprender cómo aprenden los educandos.

\section{Datos de identificación}

En este apartado se recuperan los aspectos básicos de la edad, el género y la escuela, los cuales permiten tener un panorama de los sujetos de investigación y sus características particulares. La totalidad de sujetos fue de 150 estudiantes, quienes representaban a tres subsistemas del nivel medio superior (véase tabla 3). 
Tabla 3. Subsistemas de educación media superior en el estado de Puebla

\begin{tabular}{|l|c|}
\hline Subsistema & Estudiantes \\
\hline Preparatoria BUAP & 50 \\
\hline Centro Escolar & 50 \\
\hline Colegio de Bachilleres & 50 \\
\hline & Total \\
\hline
\end{tabular}

Fuente: Elaboración propia

La presentación de resultados deriva de la elección de sujetos como una muestra no probabilística, por ser un subgrupo de la población en la que la opción de los elementos no depende de la probabilidad, sino de las características de la investigación (Hernández et al., 2014, p. 176). Por ello, se seleccionaron casos por varios propósitos, como examinar el proceso de la competencia lectora en un sitio para compararla con la de otro al describir los sistemas, procesos y resultados educativos, y así generar recomendaciones en el desarrollo de las instituciones y las prácticas educativas. El segundo dato de identificación que se solicitó fue la edad, y en este aspecto se tiene que $56 \%$ de la población cuenta con 17 años y $43.3 \%$ manifiesta 18 años de edad; además de que todos estaban en el tránsito de egresar del nivel medio superior, tal como se muestra en la tabla 4.

Tabla 4. Edad que presentan los sujetos de estudio

\begin{tabular}{|l|c|c|c|}
\hline Subsistema & 17 años & 18 años & 19 años \\
\hline Preparatoria BUAP & 33 & 16 & 1 \\
\hline Centro Escolar & 25 & 25 & 0 \\
\hline Colegio de Bachilleres & 26 & 24 & 0 \\
\hline Total & 84 & 65 & 1 \\
\hline Porcentaje & $56 \%$ & $43.3 \%$ & $0.7 \%$ \\
\hline
\end{tabular}

Fuente: Elaboración propia

En cuanto al género, la muestra de los tres subsistemas testimonia cifras más elevadas para el sexo femenino que el masculino, porque de los 150 estudiantes que participaron, 82 son mujeres y 68 son hombres, con un porcentaje de $54.6 \%$ y $45.4 \%$ respectivamente (véase figura $1)$. 
Figura 1. Género que presentan los estudiantes de bachillerato

Género

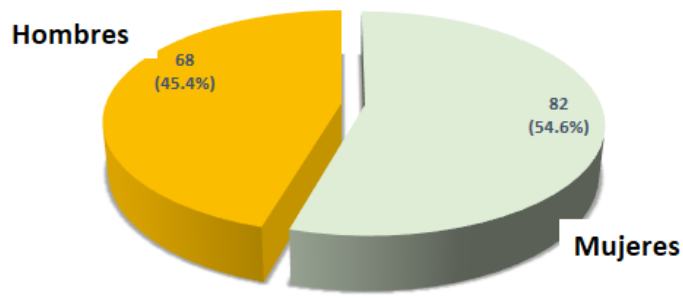

Fuente: Elaboración propia

La prueba de aptitud que se aplicó a los estudiantes de bachillerato en diferentes subsistemas consistió en un texto del cual se derivaron 17 preguntas. Estas preguntas se diseñaron atendiendo a los niveles de lectura de Kabalen y Sánchez (1997): literal, inferencial y crítico-analógico, los cuales se relacionaron con el paradigma de procesos básicos del pensamiento basado en las dimensiones cognitivas que señala la escala de estrategias de aprendizaje contextualizado (Eseac): dominio del tema, dominio de lenguajes, calidad de razonamiento, errores y su naturaleza, nivel de abstracción y conciencia cognitiva, propuesta de Juan Antonio Bernad (2000).

Los resultados obtenidos en la prueba de aptitud señalan datos relevantes con respecto a los niveles de desempeño de la competencia lectora que poseen los estudiantes. Pero antes de continuar es importante señalar que para la valoración de las respuestas se utilizó una escala Likert que midió los niveles de desempeño del I al V y ubicó los ejercicios de los participantes en las estimaciones: no suficiente, suficiente, regular, bueno y destacado (véase tabla 5).

Tabla 5. Nivel de medición para asignar puntajes a las respuestas de la prueba de aptitud

\begin{tabular}{|l|c|c|c|c|c|}
\hline \multicolumn{1}{|c|}{ Nivel } & I & II & III & IV & V \\
\hline Rango & $1.0-1.4$ & $1.5-2.4$ & $2.5-3.4$ & $3.5-4.4$ & $4.5-5.0$ \\
\hline Escala & No Suficiente & Suficiente & Regular & Bueno & Destacado \\
\hline
\end{tabular}

Fuente: Elaboración propia 


\section{Nivel de lectura literal}

Las preguntas que se formularon para esta variable midieron el nivel de desempeño de los estudiantes al realizar una lectura literal del texto, la cual implica ciertos procesos cognitivos como la observación, comparación, relación, clasificación y orden. Esta variable se integró por las preguntas:

1. De acuerdo con las características que presentó la lectura contesta qué tipo de texto es y argumenta la respuesta.

2. ¿Qué ideas o conceptos se te ocurren tras la lectura del texto?

8. Enumera las preguntas retóricas que lanza el autor y que deja abiertas; y escribe cuál es su propósito.

13. ¿Las preguntas que se presentaron en líneas anteriores las relacionas con alguna temática del área de lenguaje? Menciona cuáles.

Los resultados obtenidos en esta variable que responde al nivel literal de la competencia lectora se basan en el indicador "Dominio general del tema", con las preguntas 1, 2, 8, 13. Los estudiantes de los tres subsistemas obtuvieron de manera global una media porcentual del 2.64, lo que evidencia un desempeño regular por parte estos, al identificar en sus respuestas una serie de titubeos y debilidades en la generación del proceso lector con nivel literal, aunque en determinados referentes fueron racionales y lógicos (véase figura 2).

Figura 2. Preguntas correspondientes al nivel literal

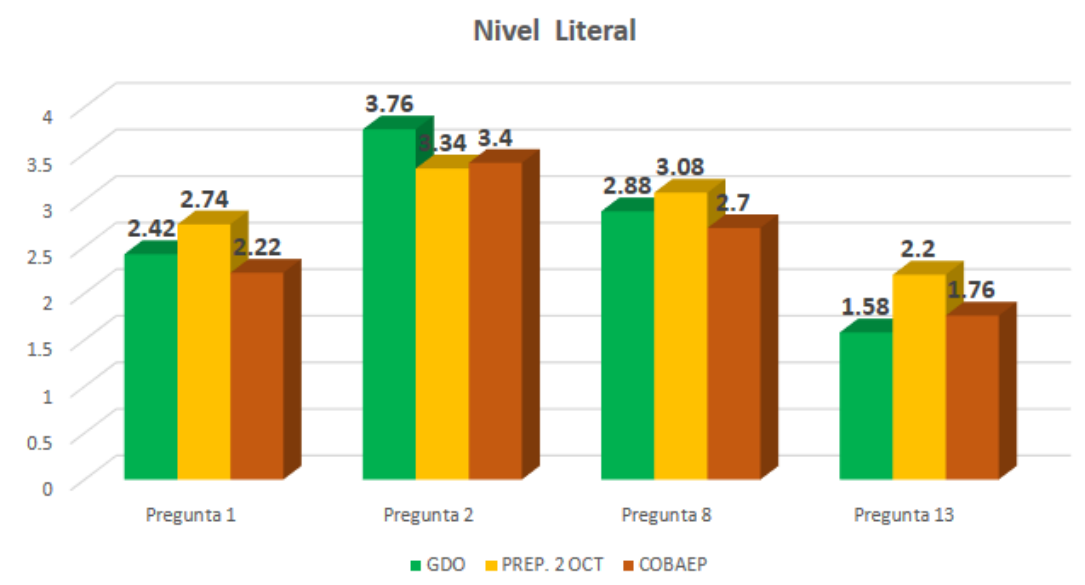

Fuente: Elaboración propia 


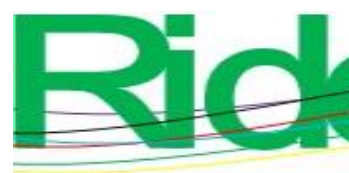

Revista Iberoamericana para la Investigación y el Desarrollo Educativo

ISSN $2007-7467$

Con respecto a las particularidades de las respuestas emitidas por los estudiantes de bachillerato, la figura 2 señala que la pregunta dos presenta una media porcentual de desempeño de nivel inferencial con valor de bueno, pero con tendencia hacia el valor de regular (3.5), porque se puede identificar que los estudiantes deducen la respuesta haciendo una relación con sus conocimientos previos, por lo que se ubica que comprenden en parte el texto de lo que no está literalmente escrito. Por otra parte, la pregunta 13 fue la de mayor dificultad para los informantes, con respecto a la relación que guarda la temática hacia el área de lenguaje a partir del texto que se les presentó; el resultado que se obtiene es una media porcentual de desempeño de nivel inferencial suficiente (1.8). En el ejercicio comparativo, los representantes de los tres subsistemas se ubican en el nivel literal con desempeño regular: Centro Escolar (2.6), Preparatoria BUAP (2.8) y Colegio de Bachilleres (2.5).

En la tarea por propiciar los niveles de lectura literal en el bachillerato es necesario asegurar el proceso de vinculación con los conocimientos previos para que los estudiantes enriquezcan la nueva información abordada y así obtengan sus propias conclusiones. Es necesario que en la educación media superior se implemente una serie de estrategias con el fin de impulsar la literacidad, que es una actividad mental importante de la competencia lectora, porque el acto de leer es un acto comunicativo y requiere de todas las capacidades del ser humano, tomando en cuenta que el abordaje de cada texto tiene su propia dinámica.

En el nivel de lectura literal se debe asegurar que el estudiante se ocupe de las ideas expuestas en el texto de modo explícito. Un señalamiento importante en relación con este nivel es que resulta erróneo considerar que el proceso de lectura es mejor en tanto más detalles se recuerden, pues la lectura es una práctica interactiva, lo cual significa que no parte únicamente del material escrito, sino que constituye una labor que se da por dos vías: del texto al lector y del lector al texto. De este modo, no se debe olvidar en el ejercicio docente que el lector selecciona aquellos datos que considera relevante para su propio proceso de lectura.

Sin duda se debe de desarrollar la comprensión e interpretación compleja de los textos en los alumnos, por lo que desde la práctica docente se deben implementar estrategias que favorezcan a las distintas etapas del desarrollo de la lectura, para que los estudiantes continúen con el proceso de volverse lectores. 


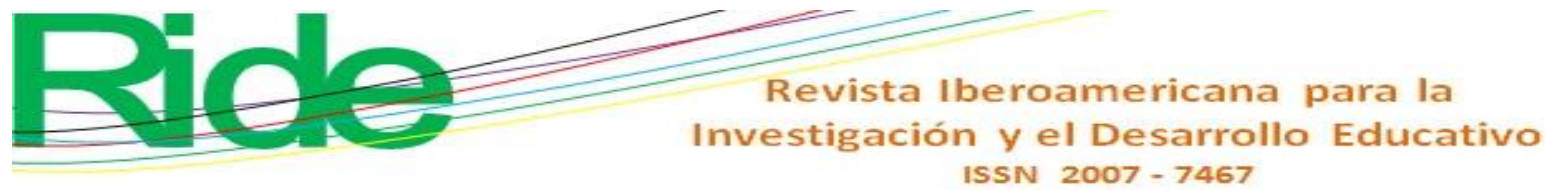

\section{Nivel de lectura inferencial}

La segunda variable es la que corresponde al nivel de lectura inferencial. Este nivel se apoya en la acciones consecuentes, derivadas y de conexión en los procesos cognitivos de los estudiantes. Aquí se interpretan una serie de mensajes implícitos, o aquellos que no se expresan directamente en el texto, y se aplican con mayor grado de complejidad a los procesos de análisis, síntesis y razonamiento, así como a la decodificación e inferencia, el razonamiento deductivo e inductivo y la adquisición de conocimiento. Las preguntas que integran esta variable son:

3. Construye un mapa conceptual del texto que leíste, pero de manera analógica relaciónalo con el uso del agua.

4. Elabora un resumen...

5. Escribe en cada línea la estructura organizacional que presenta el texto, destacando tres ideas principales que consideres por orden de importancia.

9. Encuentra tres argumentos del autor ante esta problemática social.

10. Elabora un cuadro evaluador de las situaciones positivas y negativas que se realizan en ambos países.

14. Explica qué pregunta te ha resultado más fácil de contestar, anota su número y argumenta el por qué.

15. Explica qué pregunta te ha resultado más difícil de contestar, anota su número y argumenta el por qué.

17. Dibuja un ícono que represente el cuidado de los bosques o el respeto a los árboles.

Los resultados obtenidos en esta variable, que responde al nivel inferencial, se basan en tres indicadores: "Dominio de lenguajes", con las preguntas 3, 4, 17; "Calidad de razonamiento", que incluye las preguntas 5, 9, 10; y "Error y su naturaleza", que vincula las preguntas 14 y 15. En el nivel inferencial de la competencia lectora, de acuerdo con las respuestas que proporcionan los estudiantes de los tres subsistemas, se obtuvo de manera global una media porcentual del 2.82, lo que evidencia un desempeño regular al identificarse en sus respuestas una serie de titubeos y debilidades para demostrar el manejo la lectura con el nivel inferencial (véase figura $3)$. 


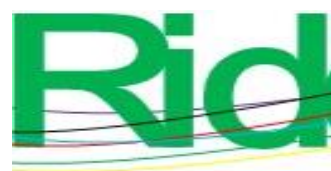

Revista Iberoamericana para la Investigación y el Desarrollo Educativo ISSN $2007-7467$

Figura 3. Resultados del nivel inferencial

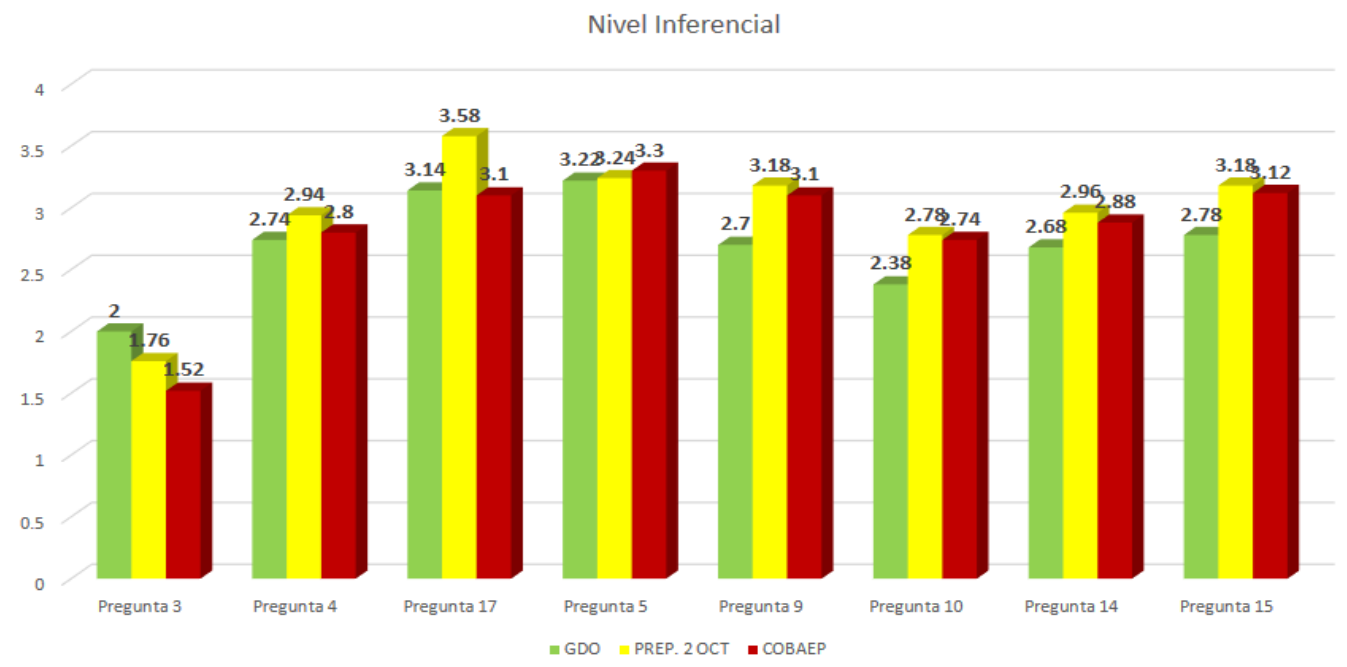

Fuente: Elaboración propia

El nivel inferencial demanda la elaboración de interpretaciones e hipótesis por parte del lector, porque debe preponderar el significado del texto, y para cada uno se deben aprender aquellas categorías que verifiquen una situación relevante en la generación del significado (Méndez, 2006, p. 148).

La figura 3 indica que las respuestas proporcionadas por los informantes en los reactivos 5 y 17 señalan una media porcentual de desempeño regular del nivel inferencial (3.2). Cabe subrayar que los estudiantes deducen la respuesta haciendo una relación con sus funciones de estructura organizacional y el dominio de su lenguaje icónico, lo que les permite destacar ideas principales por consideración en orden de importancia, así como la representación a manera de dibujo de las ideas que se comprenden posterior a la lectura.

Por otra parte, la pregunta tres es la que mayor dificultad registró en los desempeños inferenciales de los estudiantes de bachillerato. El resultado que se obtuvo fue una media porcentual de escala suficiente (1.76). Las inconsistencias se detectaron en la construcción de un mapa conceptual del texto que acompañó a la prueba de aptitud, cuando a los sujetos informantes se les solicitó que por analogía relacionaran el organizador gráfico con un contenido específico que debieron identificar desde el texto, en este caso el uso del agua. En el ejercicio comparativo, los estudiantes de los tres subsistemas se ubicaron con un desempeño regular: Centro Escolar (2.7), Preparatoria BUAP (2.9) y Colegio de Bachilleres (2.8). 


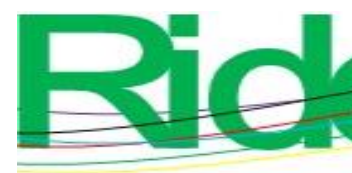

Revista Iberoamericana para la Investigación y el Desarrollo Educativo

ISSN $2007-7467$

Las concepciones del nivel de lectura inferencial señalan un procedimiento alternativo entre la comprensión que está dirigida conjuntamente por los datos explícitos del texto y por el conocimiento preexistente en el estudiante lector. Por ello, el significado del texto está solo parcialmente explícito. La lectura debe ser un proceso constructivo e inferencial, caracterizado por la formación y confirmación de hipótesis acerca de lo que trata el texto, de ahí que se adopten esquemas de conocimiento como referentes explicativos, considerados estos como dimensiones altamente estructuradas con las que se organiza el conocimiento y cómo este debe ser usado. Contienen un conjunto de variables interrelacionadas entre sí y que no toman valores fijos sino prototípicos.

El nivel de lectura inferencial activa en los estudiantes, y en función del contexto y la actividad principal, la determinación de un esquema dado que proporciona una interpretación adecuada a la situación presentada en la lectura. Desde la competencia lectora por nivel inferencial se deben asegurar diversos procesos lectores basados en la función perceptiva, de comprensión y resolución de una serie de problemas que se logran identificar.

El significado no reside en las palabras, ni en las frases, ni en los párrafos, ni siquiera en el texto considerado globalmente, sino más bien corresponde al estudiante lector, que puntualmente representa la información del texto y la ajusta a su contexto, conocimiento sobre el mundo y a sus propósitos de comprensión en un momento específico, todo lo cual apela a sus dominios lingüísticos, situacionales, actitudinales, entre otros.

\section{Nivel de lectura crítico-analógico}

Este proceso se basa en el razonamiento analógico, por lo que se espera que el estudiante aplique los niveles de lectura literal e inferencial y, con base en ello, interpretar la temática del escrito para establecer relaciones analógicas de diferente índole. El estudiante debe relacionar los hechos que ocurren en la lectura con otros acontecimientos que suceden en su vida diaria presente, pasada o futura; asimismo, establecer relaciones entre una lectura y otra. En este nivel, se formulan juicios críticos acerca de lo leído y se emiten opiniones o posiciones con respecto a su escala axiológica, porque es capaz de formular sus propias conclusiones. En total se formularon cinco preguntas: 


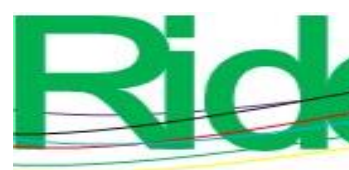

\section{Revista Iberoamericana para la Investigación y el Desarrollo Educativo \\ ISSN $2007-7467$}

6. ¿Consideras que el autor del texto "Bosques" tiene un objetivo en particular? Argumenta tu respuesta.

7. ¿El contenido de este texto lo relacionaste en algún momento con situaciones de tu vida cotidiana? Menciona dos ejemplos o situaciones.

11. Menciona por qué crees que en nuestro país no hay programas de reforestación.

12. En tu opinión, ¿a corto o mediano plazo México podrá contar con un programa efectivo de reforestación? Argumenta tu respuesta.

16. ¿Cómo has hecho para darte cuenta de lo que tenías que responder ante los cuestionamientos que se te presentaron?

Los resultados obtenidos en esta variable, que responde al nivel de lectura críticoanalógico, aluden a tres indicadores: “Abstracción”, con las preguntas 6, 7, 11; "Calidad de razonamiento", con las preguntas 5, 9, 10; y "Conciencia cognitiva", con las preguntas 12, 16. En el nivel crítico-analógico de la competencia lectora, de acuerdo con las respuestas que proporcionan los estudiantes de los tres subsistemas, se obtuvo de manera global una media porcentual de 2.92, el resultado más alto que se produce al codificar la totalidad de la prueba; aun así, dicha media apunta a un desempeño regular: los alumnos manifestaron titubeos en la generación del proceso de lectura de nivel crítico-analógico (véase figura 4).

Figura 4. Resultados del nivel crítico

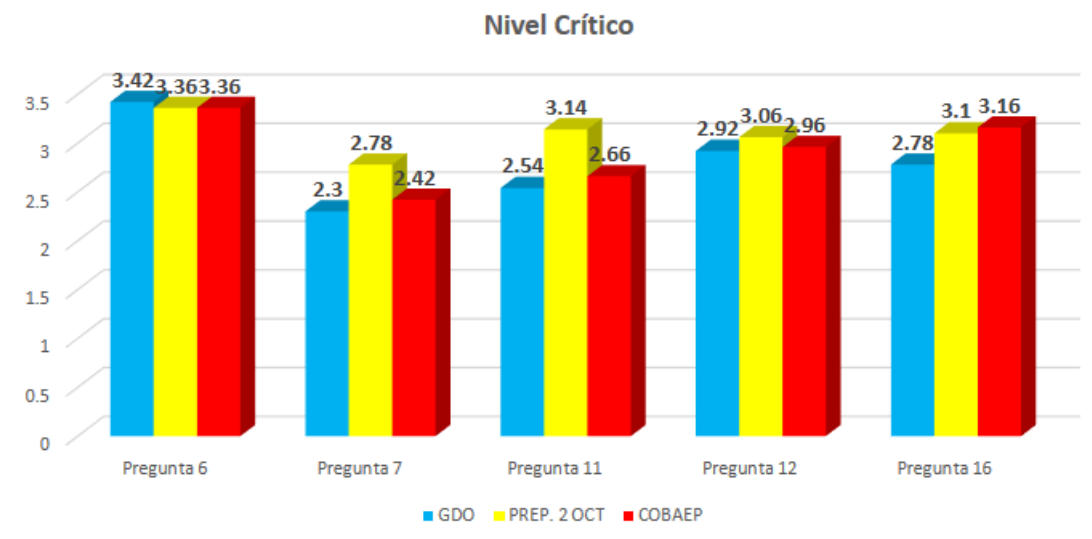

Fuente: Elaboración propia

En el nivel crítico-analógico se debe desarrollar la condición metacognitiva del aprendizaje de la lectura, porque es la conciencia que tiene el aprendiz para actuar en el procesamiento de la información que arroja la lectura. Los estudiantes de bachillerato, desde el nivel crítico-analógico, alcanzaron el puntaje más alto con respecto a sus desempeños en la 


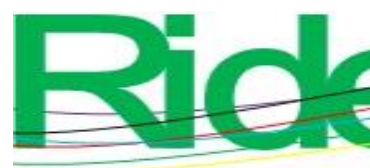

Revista Iberoamericana para la

Investigación y el Desarrollo Educativo

ISSN $2007-7467$

competencia lectora y exteriorizan un nivel aceptable de conocimiento sobre el uso de las estrategias, sobre todo crítica para leer y comprender con precisión; fueron además competentes en la demostración de su dominio de abstracción y de conciencia cognitiva (Calero, 2011).

La figura 4 señala que la pregunta seis presenta una media porcentual de desempeño de nivel crítico-analógico con tendencia a un nivel regular (3.4). Se puede observar que los estudiantes regulan los procesos cognitivos de la lectura a través de las estrategias de control de los progresos en la comprensión lectora de los diferentes tipos de textos. Por otra parte, la pregunta siete es la que mayor problema generó en los informantes con respecto a la relación que guarda la temática hacia el área de lenguaje a partir del texto que se les presentó. El resultado obtenido es una media porcentual de desempeño de nivel inferencial regular con tendencia hacia el suficiente (2.5). En el ejercicio comparativo, los estudiantes de los tres subsistemas se ubican en el nivel crítico-analógico con desempeño regular: Centro Escolar (2.8), Preparatoria BUAP (3.1) y Colegio de Bachilleres (2.9).

En la variable de nivel de lectura crítico-analógico, la muestra de estudiantes en general tuvo un desempeño aceptable con respecto al proceso lector de centrar la atención en las ideas principales del texto, la coherencia en la expresión de opiniones y la compatibilidad con sus conocimientos previos. El estudiante de bachillerato comprende que, con los textos, puede modificar sus esquemas del conocimiento al concurrir con otros nuevos que la lectura le ofrece, dado que el significado está solo parcialmente determinado por el texto en sí. La competencia lectora desde el nivel crítico-analógico impulsa estructuras de conocimiento que el estudiante construye para comprender el mundo que le rodea y los aprendizajes que debe adquirir.

\section{Discusión}

Existen varios niveles de comprensión: se puede leer las líneas (la acción literal), entre líneas (la acción implícita) y detrás de las líneas (la ideología o el punto de vista, los valores y los presupuestos). Por lo que hoy en día es importante lograr armonizar la totalidad de ellos como parte de la competencia lectora, porque, cuando se comprende un texto, en el estudiante se activa el sistema cognitivo que le permite valorar y actuar en una situación compleja. Desde la lectura literal, inferencial y crítico-analógica, el alumno de bachillerato cuenta con la capacidad para generar representaciones mentales que constituyen la base de su funcionamiento, como es el caso 


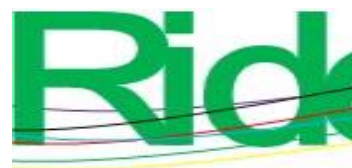

Revista Iberoamericana para la
Investigación y el Desarrollo Educativo
ISSN $2007-7467$

de la competencia lectora, que resulta pertinente en el contexto contemporáneo porque es objeto de atención en los sistemas educativos en los ámbitos regionales, nacionales e internacionales.

La lectura en sus diferentes niveles es la herramienta base para que se produzcan futuros aprendizajes, por lo que es algo que se inicia en la formación de los primeros años de la escolarización, y que longitudinalmente va evolucionando hacia un conjunto de competencias comunicativas que se construyen como estrategias y desarrollan a lo largo de la vida en los diversos contextos sociales, en la misma interacción con las personas que nos relacionamos.

La lectura y la escritura en los estudiantes de bachillerato otorgan múltiples beneficios: la adquisición de la mayoría del conocimiento y el fortalecimiento de la cultura. Particularmente, desde la competencia lectora se transfieren conocimientos de una generación a otra, y se promueve la sensibilidad del educando al proporcionarle diversas herramientas para su comunicación y dominio del lenguaje verbal, icónico y analógico. El buen desarrollo de la competencia lectora es uno de los elementos que aumenta la probabilidad de acceder a un escenario social con mayor oportunidad; por ello, es importante valorar la competencia lectora del estudiante de educación media superior al ser una de las acciones clave en su formación escolar y social.

El desarrollo de estrategias para propiciar la competencia lectora requiere por parte de los docentes en bachillerato no solo del conocimiento, sino de la reflexión, razonamiento y aplicación consciente de las mismas, además del manejo de información de otras asignaturas y vinculación con los ámbitos culturales que permitan comunicarse. Esto implica que los métodos de enseñanza sean más activos, incluyentes y colaborativos, aunado de la aplicación de diferentes metodologías que potencien el desarrollo de la competencia lectora. 


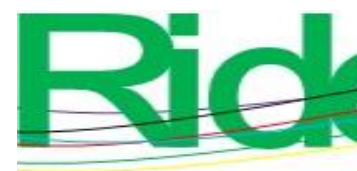

Revista Iberoamericana para la Investigación y el Desarrollo Educativo

ISSN $2007-7467$

\section{Conclusiones}

Si se pone a los estudiantes de bachillerato en la necesidad de leer, se puede fomentar en ellos la lectura por motivación, por lo que se debe poner a disposición de los jóvenes textos literarios que respondan a sus gustos y preferencias. Con el uso de estrategias para fomentar la comprensión lectora, se va logrando paulatinamente el desarrollo de capacidades cognitivas que les permitan a los jóvenes leer, comprender y apropiarse del texto. Como tarea educativa queda claro que esta no es exclusiva de las asignaturas que conforman el campo disciplinario del lenguaje y la comunicación, sino que se extiende a las demás asignaturas que integran los mapas curriculares en el bachillerato.

Se recomienda para los subsistemas de educación media superior en el estado de Puebla que impulsen proyectos de lectura que involucren a docentes y estudiantes para que aprovechen los acervos bibliográficos, así como que se cuiden las iniciativas de la promoción de los espacios lectores en los bachilleratos para que no se conviertan en un requisito más del sistema educativo. Se trata de propiciar una serie de actitudes en los estudiantes para que compartan momentos de consulta, investigación y de lectura con propósitos muy diversos, favoreciendo la interacción y el intercambio de ideas.

Se sugiere a los docentes de bachillerato que en la realización de los procesos de enseñanza en el aula no pasen por alto los conocimientos previos de sus educandos, que pongan atención en aquellas actividades que se realizan antes de leer para ayudar a los jóvenes a encontrarse con el texto desde perspectivas más acordes con la intencionalidad de la lectura, para aumentar su vocabulario, plantearse palabras claves que aparecen en la lectura, al igual que generar en el alumno la búsqueda activa del significado de la lectura.

Otra recomendación para el profesor de bachillerato es dar a conocer a los alumnos los propósitos por los cuales se lee, con la intención de generar una intuición emotiva a la hora de abordar la lectura, la cual se incrementa si el docente los motiva y les hace ver la utilidad y pertinencia de la lectura. Se trata de que el alumno lector se encuentre en un estado volitivo para comprender lo que lee. Es recomendable también poner a disposición de los estudiantes todos aquellos bagajes de palabras, sinónimos, antónimos, analogías, como herramientas que pueden utilizar para comprender mejor un texto y sacarle todo el provecho posible para fortalecer así la competencia lectora. 


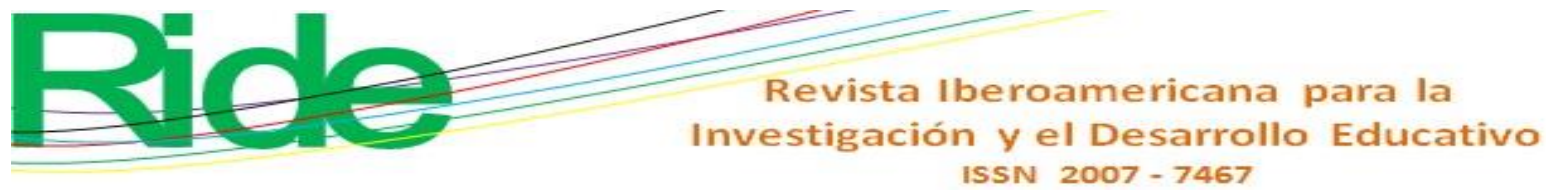

\section{Referencias}

Bernad, J. (2000). Modelo cognitivo de evaluación educativa. Escala de estrategias de aprendizaje contextualizado (ESEAC). Madrid, España: Narcea.

Calero, A. (2011). Cómo mejorar la comprensión lectora. Estrategias para lograr lectores competentes. España: Wolters Kluwer.

Escamilla, A. (2008). Las competencias básicas. Barcelona, España: Graó.

Ferreiro, E. (2002). Pasado y presente de los verbos leer y escribir. Buenos Aires, Argentina: Fondo de Cultura Económica.

Ferreiro, E. y Gómez, M. (2002). Nuevas perspectivas sobre los procesos de lectura y escritura. México: Siglo XXI Editores.

Gómez, M. (1996). La lectura en la escuela. México: SEP.

Goodman, K. (1982). El proceso de lectura: consideraciones a través de las lenguas y del desarrollo. En Ferreiro, E. y Gómez, M. (comps.), Nuevas perspectivas sobre los procesos de lectura y escritura (pp. 13-28). México: Siglo XXI Editores.

Hernández, R., Fernández, C. y Baptista, P. (2014). Metodología de la investigación. México: McGraw-Hill.

Hymes, D. (1971). Competence and performance in linguistic theory. Acquisition of languages: Models and methods. New York, United States: Academic Press.

Instituto Nacional para la Evaluación Educativa [INEE] (2017). México en PISA 2015. México: Instituto Nacional para la Evaluación Educativa.

Kabalen, D. y Sánchez, M. (1997). Lectura analítica-crítica. México: Trillas.

Méndez, S. (2006). Comprensión lectora y textos literarios: Una propuesta psicopedagógica. Revista Educación, 30(1), 141-155.

Organización para la Cooperación y el Desarrollo Económicos [OCDE]. (2006). PISA 2006. Marco de la evaluación. Conocimientos y habilidades en Ciencias, Matemáticas y Lectura. Madrid, España: Santillana Educación.

Secretaría de Educación Pública [SEP]. (21 de octubre de 2008). Acuerdo número 444 por el que se establecen las competencias que constituyen el marco curricular común del Sistema Nacional de Bachillerato. Diario Oficial de la Federación. Recuperado de http://www.sems.gob.mx/work/models/sems/Resource/10905/1/images/Acuerdo_444_ma rco_curricular_comun_SNB.pdf. 


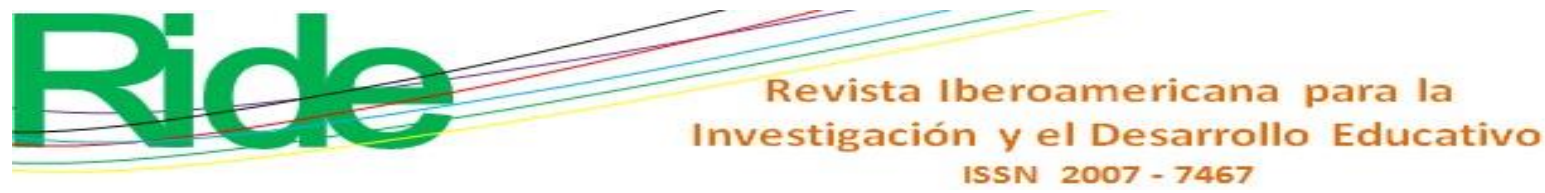

Secretaría de Educación Pública [SEP]. (2017). PLANEA. Resultados nacionales 2017. México:

Secretaría

de

Educación

Pública.

Recuperado

de

http://planea.sep.gob.mx/content/general/docs/2017/ResultadosNacionalesPlaneaMS2017. PDF. 